\title{
Surgical approach to synchronous colorectal liver metastases: staged, combined, or reverse strategy
}

\author{
Heather A. Lillemoe, Jean-Nicolas Vauthey \\ Department of Surgical Oncology, The University of Texas MD Anderson Cancer Center, Houston, TX, USA \\ Contributions: (I) Conception and design: All authors; (II) Administrative support: JN Vauthey; (III) Provision of study material or patients: All \\ authors; (IV) Collection and assembly of data: All authors; (V) Data analysis and interpretation: All authors; (VI) Manuscript writing: All authors; (VII) \\ Final approval of manuscript: All authors. \\ Correspondence to: Jean-Nicolas Vauthey, MD. Department of Surgical Oncology, The University of Texas MD Anderson Cancer Center, 1515 \\ Holcombe Boulevard, Unit 1484, Houston, TX 77030, USA. Email: jvauthey@mdanderson.org.
}

\begin{abstract}
An increasing number of patients with colorectal cancer (CRC) are presenting with synchronous disease to the liver. The optimal surgical approach for this complex patient group is controversial, but ultimately depends on individual patient characteristics and institutional practices. Surgical strategies include the traditional staged approach, a combined colorectal and liver resection, or a liver-first reverse approach. In this review, the authors will provide an overview of each strategy, including case examples demonstrating the benefits of the more recently described liver-first approach, while arguing for individualized planning and multidisciplinary discussion for every patient.
\end{abstract}

Keywords: Synchronous colorectal liver metastases (SCRLM); liver-first reverse approach; multidisciplinary discussion

Submitted Apr 02, 2019. Accepted for publication May 23, 2019.

doi: $10.21037 / \mathrm{hbsn} .2019 .05 .14$

View this article at: http://dx.doi.org/10.21037/hbsn.2019.05.14

\section{Introduction}

Colorectal cancer (CRC) is the third most common malignancy worldwide and as of 2018 , it is the second leading cause of cancer death (1). The liver is the most common site of metastatic disease, with an estimated $15-25 \%$ of CRC patients presenting with synchronous colorectal liver metastases (SCRLM) $(2,3)$. It is important to distinguish between synchronous disease, or liver metastases diagnosed prior to or at the time of diagnosis of CRC, and metachronous disease, or liver metastases diagnosed after resection of the primary tumor, given that recent literature suggests the incidence of SCRLM is increasing compared to metachronous (4-6). This is likely due to improvements in imaging technology allowing for earlier diagnosis. The definition of synchronous disease varies in the surgical literature in regards to the timing of liver disease diagnosis in relationship to the diagnosis of the primary tumor, ranging from 0 all the way to
12 months. In agreement with the international multidisciplinary consensus published in 2015, the authors suggest standardized definitions must be adopted. For the purpose of this paper, we will refer to patients presenting with imaging indicating a presence of both colorectal and liver disease (in the absence of other metastases) (6). Currently, synchronous disease is associated with a less favorable prognosis than metachronous disease $(6,7)$.

For patients with colorectal liver metastases (CRLM), surgical resection is essential for long-term survival $(8,9)$ and results in 5 -year overall survival (OS) rates as high as $58 \%(9,10)$. However, the majority of patients who present with SCRLM have initially unresectable disease (6). Preoperative chemotherapy is used to treat the advanced stage, prevent further progression, and to improve patient selection for liver-directed surgical therapy. Thus, a thorough and multidisciplinary approach that includes early surgical consultation is imperative $(6,11)$. In this review, the authors will discuss the treatment strategies for patients 
presenting with SCRLM. These include: the traditional staged approach, the simultaneous combined approach, or the liver-first reverse approach.

\section{A historical review: traditional staged approach to SCRLM}

The traditional treatment algorithm for SCRLM is a staged approach that includes primary tumor resection, followed by systemic chemotherapy then resection of liver metastases for patients without progression of disease. The benefits of this approach are thought to include ensured treatment of the primary tumor (both to reduce the risk of new metastatic disease and to avoid the development of complications such as obstruction, perforation, or bleeding) and selection of patients with optimal tumor biology (often via extended pre-hepatectomy chemotherapy). Proponents of the traditional approach may also argue that the morbidity of simultaneous colorectal and liver resection is prohibitive (12).

A major concern with the traditional staged approach is that it ignores the fact that prognosis is driven by the metastatic disease. By definition, patients with SCRLM have systemic disease and by delaying both chemotherapy and liver resection, the risk of unresectability only increases. Should a patient experience a complication after resection of the primary tumor, such as anastomotic leak or wound infection, significant delays in treatment of the systemic disease can be incurred. Additional counterarguments are made against the need for upfront colorectal tumor treatment to avoid symptoms of obstruction, perforation, or bleeding. Retrospective studies comparing asymptomatic patients with SCRLM who did or did not undergo primary tumor resection have shown that the risk of developing such symptoms is negligible (13-16). A prospective, multicenter study using preoperative mFOLFOX6 with bevacizumab for patients with asymptomatic colon cancer and unresectable metastatic disease demonstrated acceptable rates of primary tumor-related complications (14\%) (17). Further, as many as $30 \%$ of patients who do undergo elective colorectal resection will have a subsequent complication $(15,18)$, suggesting that upfront resection of the primary tumor is more likely to cause complications than prevent them. Studies comparing groups with similar metastatic burden have found no difference in median survival based on patients who do or do not undergo colorectal resection for CRC in the setting of unresectable metastatic disease $(15,18)$.
An additional argument against the traditional approach lies in the advantage of early chemotherapy. Along with its survival benefits, early treatment with chemotherapy can translate to increased rates of resectability for unresectable CRLM. In 1996, Bismuth et al. reported a 16\% conversion rate from unresectable to resectable metastatic liver disease with survival rates of $40 \%$ at 5 years (19). Since that time, experts agree that the use of preoperative chemotherapy is indicated for unresectable CRLM, leading to complete resection in $15-30 \%$ of patients $(12,20)$. Thus, particularly in the setting of unresectable liver disease, delay in its initiation should be avoided. Recommended regimens include those used for advanced CRC, such as triplet therapy or doublets with bevacizumab or anti-EGFR therapy (21-23). While the issue of preoperative chemotherapy for initially resectable CRLM is more controversial, it is still recommended by most for synchronous disease (6). Results of a randomized controlled trial comparing perioperative FOLFOX (6 cycles before and 6 cycles after surgery) to surgery-only in initially resectable CRLM found improved progression-free survival with perioperative chemotherapy, although no significant difference in OS was shown with longer-term follow-up $(24,25)$. These results do not directly translate to the SCRLM population, given only one-third of the patients presented with synchronous disease. However, given the high rates of recurrence for metastatic CRLM, there is rationale for perioperative chemotherapy in this disease, with mixed results shown in the literature (26-28). Based on the National Comprehensive Cancer Network (NCCN) guidelines, a total of six months perioperative treatment is preferred (29). Prolonged administration of chemotherapy should be avoided given the risk of chemotherapy-associated liver injury (30-32).

\section{Combined approach to SCRLM}

The combined approach to SCRLM involves complete surgical resection of liver metastases at the time of primary colorectal resection. This is most frequently preceded by preoperative systemic chemotherapy. Obvious benefits of the combined approach are efficient and early treatment of all disease with only one operation requiring general anesthesia. Most typically, patients selected for simultaneous resection have tumors that are easier to approach operatively: smaller or fewer liver tumors and right-sided colonic tumors. Aside from strict patient selection, downsides to approaching both the primary colorectal and liver disease in one operation are the 


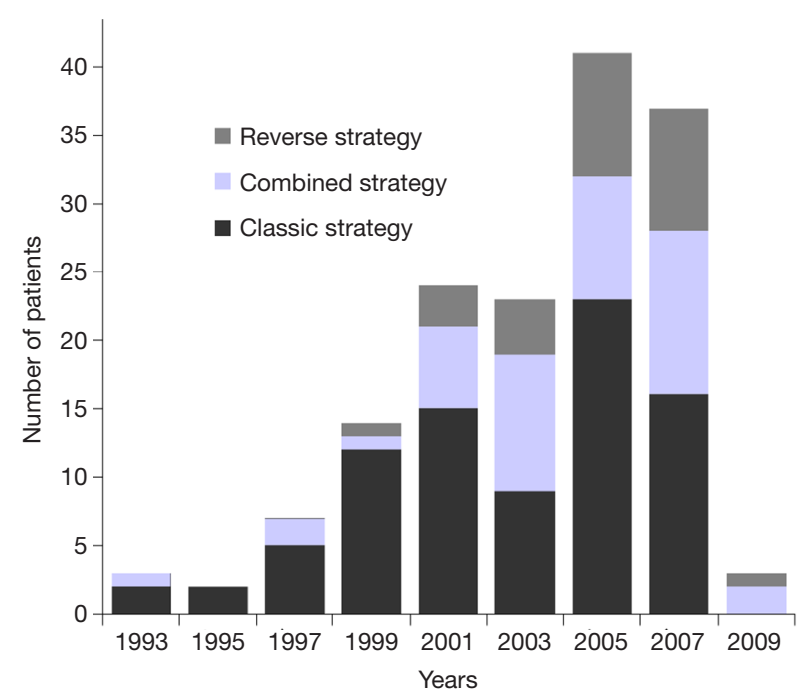

Figure 1 Patients operated on for synchronous liver metastases at MD Anderson Cancer Center over time. With permission from Brouquet et al. 7 Am Coll Surg, 2010.

prolonged operative time and potentially increased risk of perioperative morbidity. Particular attention to bleeding risk and intestinal perfusion preoperatively is essential in order to determine what types of cases can be performed simultaneously. In patients initially scheduled for combined resection, we make the intraoperative decision not to proceed with resection of the primary in cases of unanticipated difficult or protracted liver resection.

A number of early surgical series reported outcomes after using a combined approach to SCRLM (33-36). Some authors reported higher operative morbidity and mortality associated with simultaneous resection, leading them to recommend the traditional staged approach $(33,34,37)$. In a study by Martin et al., 134 patients who underwent combined resection of CRLM and a primary colorectal tumor were compared to 106 patients who underwent staged resections. Patients with right-sided colon tumors, and smaller and fewer liver metastases undergoing less extensive liver resection, were more likely to undergo a simultaneous approach. The combined group had fewer complications $(49 \%$ vs. $67 \%, \mathrm{P}<0.003)$ and had a shorter median hospital length of stay (10 vs. 18 days, $\mathrm{P}=0.001$ ), with similarly low mortality rates (38). A large multicenter study by Reddy et al. included 327 patients requiring major liver resection for SCRLM. Patients who underwent simultaneous colorectal resection and major hepatectomy $(\mathrm{n}=36)$ had higher rates of severe morbidity (36\% vs. $15 \%$,
$\mathrm{P}<0.05)$ and mortality $(8 \%$ vs. $1 \%, \mathrm{P}<0.05)$ compared to the staged approach $(n=291)$ (39). Therefore, the authors recommend that caution be exercised when considering a combined approach to SCRLM for cases requiring a major liver resection.

\section{Liver-first approach to SCRLM}

More recently, the liver-first or reverse strategy has been proposed to treat SCRLM. In this treatment algorithm, preoperative chemotherapy is administered prior to hepatectomy and followed by resection of the colorectal primary at a later date. Initially proposed in 2006, Mentha et al. described the feasibility of this approach and impressive survival outcomes in 20 patients with advanced disease (40). The approach proved particularly wellsuited for rectal cancers, with $7 / 8$ patients with rectal tumors receiving a full course of pelvic radiotherapy shortly after hepatectomy but prior to rectal surgery. A notable advantage of this strategy, as well as the combined strategy, is that the delay in addressing the systemic disease that drives OS is avoided. Whether this is with initial locoregional therapy of a primary rectal tumor or surgical resection, both can result in significant delays in initiation of systemic chemotherapy. Critics of the reverse approach argue that failure to treat the primary colorectal tumor will lead to complications such as bleeding, obstruction, or perforation. However, as previously mentioned, rates of primary tumor-related complications in asymptomatic patients treated with chemotherapy are quite low (13-16).

Brouquet et al. compared the three strategies for managing SCRLM, analyzing 72 staged, 43 simultaneous, and 27 reverse approach cases (3). In this study out of a single, large comprehensive cancer center, not only did the number of patients undergoing treatment for SCRLM increase over time, but there was also a shift in the preferred surgical strategy, with more patients undergoing combined or reverse approaches in more recent years (Figure 1). Patients who underwent the combined approach were less likely to receive six or more cycles of preoperative chemotherapy $(\mathrm{P}<0.001)$. Those who underwent the reverse approach were much more likely to receive preoperative bevacizumab (78\%) compared to the staged and combined groups $(31 \% ; \mathrm{P}<0.001)$. Patients treated with the reverse approach were also more likely to have a rectal primary tumor and a higher number of CRLMs resected (median of 4). Among 41 patients intended for the reverse strategy, $14(34 \%)$ did not have resection of the primary tumor for 
reasons such as: progression of metastatic disease (64\%), complete response of a rectal primary tumor (14\%), and postoperative death after liver resection, progression of primary tumor, or loss of follow-up in one patient each. Two of the 41 patients (5\%) had symptoms from their primary tumor requiring colostomy. The mortality and cumulative morbidity rates between each of the three strategies were comparable. Survival rates also did not significantly differ. Multivariable analysis showed that greater tumor size $(>3 \mathrm{~cm})$ and cumulative postoperative morbidity were independently associated with survival.

\section{Recommendations for approaching SCRLM}

\section{Preoperative considerations \& strategies for complex cases}

Patients with SCRLM are not always candidates for upfront surgical resection given the overall burden of disease (6). As previously mentioned, preoperative chemotherapy can improve resectability in many cases $(12,19,20)$. Imaging characteristics such as tumor size [based on traditional Response Evaluation Criteria in Solid Tumors (RECIST) criteria] and tumor morphology should be used to determine response to preoperative therapy (6). Morphologic response of tumor attenuation, degree of enhancement, and borders on computed tomography (CT) imaging has been shown to correlate with pathologic response and survival outcomes in patients receiving preoperative chemotherapy regimens containing bevacizumab (41). Follow-up studies suggest that morphologic response may be superior to RECIST criteria for assessing pathologic tumor response in CRLM regardless of the preoperative regimen $(42,43)$. In addition to tumor-specific characteristics, it also important to assess for signs of steatosis and portal hypertension on preoperative imaging. Approximately $10 \%$ of patients receiving preoperative chemotherapy for CRLM develop sinusoidal obstruction syndrome and up to $20 \%$ develop steatosis $(30,44)$. Liver injury varies depending on the type and duration of chemotherapy (45).

In cases of insufficient future liver remnant (FLR), strategies such as portal vein embolization (PVE) can be effective. During PVE, the portal venous system draining the affected liver (planned for resection) is embolized in order to induce hypertrophy of the non-embolized liver and reduce the risk of post-hepatectomy liver failure (45). The two-stage approach to hepatectomy can be used, often in conjunction with PVE, as a sequential treatment strategy in cases of bilateral CRLM. Currently, the most commonly employed strategy is as follows: first-stage liver resection intended to "clear" the less-involved hemi-liver, followed by PVE within 2-5 weeks of the first stage, and second stage hepatectomy after another 5-8 weeks (46). In some cases, combined colorectal tumor resection can be performed at the time of one of the two liver resections. This two-stage approach to CRLM has been shown to be safe and effective, with completion rates ranging from 60-90\% (46-50). Associated liver partition and portal vein ligation for staged hepatectomy (ALPPS) is an additional strategy that has been used in setting of advanced bilateral CRLM. Currently there is no data evaluating the appropriate sequencing for the management of SCRLM in association with ALPPS.

\section{Preoperative molecular and pathologic tumor assessment}

While achieving a negative surgical margin should remain the goal of CRLM resection (with at least $1 \mathrm{~mm}$ clearance), recent investigations have emphasized the importance of pathologic response to preoperative therapy and molecular tumor biology (6). While complete pathologic response is rare, pathologic assessment for tumor response to preoperative chemotherapy is essential for guiding further therapy and understanding prognosis $(51,52)$. In addition, RAS mutation has been shown to be associated with worse survival outcomes after resection of CRLM (53-55). A metaanalysis of studies analyzing the prognostic impact of KRAS by Brudvik et al. found a mutation rate of $28 \%$ and a negative association with OS (hazard ratio 2.24, 95\% confidence interval: 1.76-2.85) (56). BRAF V600E mutation, although more rare, is also associated with poor OS (57-59). Tumor biology and pathologic assessment should be incorporated into multidisciplinary discussions whenever possible.

\section{An individualized approach}

Ultimately, comparing the staged, combined, and reverse strategies to SCRLM is based on retrospective surgical series with inherent selection bias as patients are chosen for each approach based on individual characteristics. Similarly, improvements in technology and perioperative care limit direct comparison of an open-incision staged approach performed ten years ago to a simultaneous, often minimallyinvasive, approach to SCRLM today. Undoubtedly, the patients undergoing these operations will have differences in their presentation, tumor biology, and metastatic burden of disease. Institutions and disciplines also have varying opinions regarding best practices. Without a randomized 


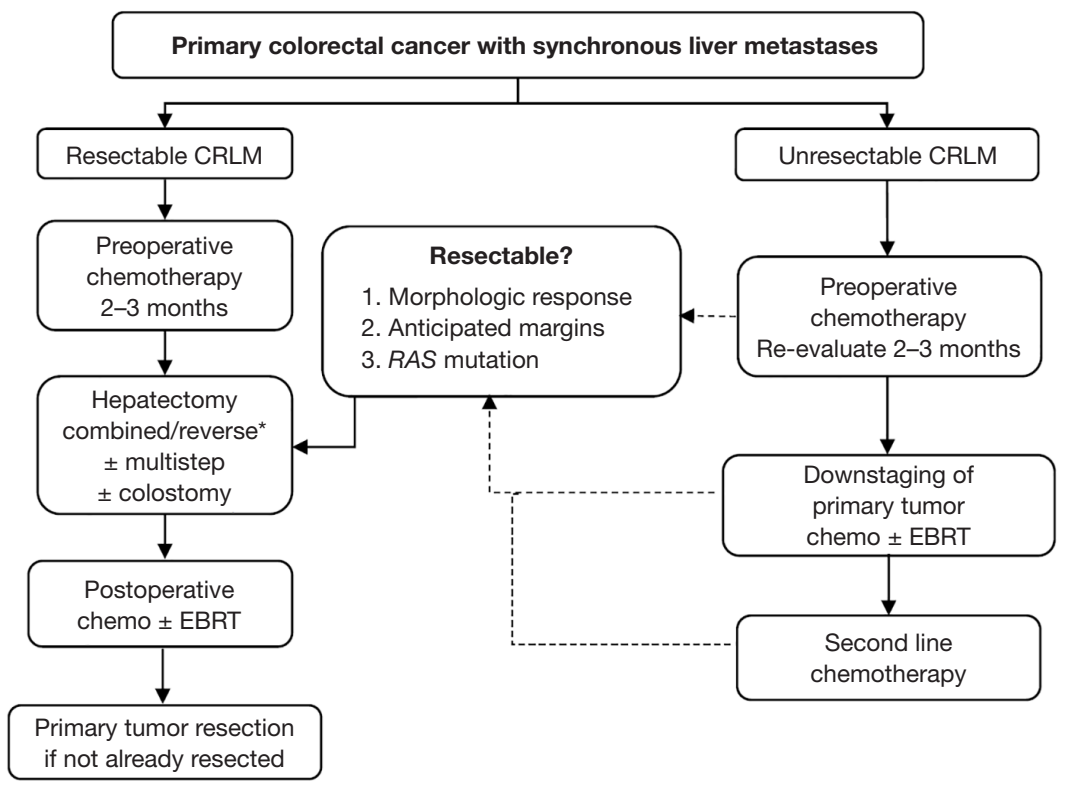

Figure 2 Structured approach to synchronous colorectal liver metastases (SCRLM). *, this is provided there is no obstruction as determined by preoperative colonoscopy as described in discussion section: "An Individualized Approach." CRLM, colorectal liver metastases.

controlled trial, which would be impossible to perform given the large spectrum of patient presenting with SCRLM, the authors can only use experience and results of the published surgical literature to make recommendations. Figure 2 shows a structured treatment algorithm used at our institution, which can be referenced when dealing with SCRLM. Importantly, an individualized approach must be used for every patient.

There are three major preoperative issues to consider when first evaluating a patient with SCRLM. First, the extent of surgery will have a significant impact on choosing the appropriate strategy. For example, an abdominoperineal resection for rectal cancer may be combined with a wedge resection of the liver, but it would not be appropriate to combine it with a right hepatectomy. Second, the wide spectrum of patients and variability in presentation mandate specific considerations. Patient-specific risk factors need to be weighed when considering a combined approach. A higher-risk combined operation (such as a right colectomy and right hepatectomy) may be undertaken, permitting the patient is low-risk without major comorbidities and a normal body habitus. As mentioned, close collaboration with all surgeons and anesthesiologists involved in the operation is essential to discuss the sequence of resection (colon or liver first), specific perioperative needs (such as low central venous pressure for liver resection), or changes in the risk of the operation than may preclude a planned combined procedure. The authors recommend starting with liver resection and continuing with the colon or rectal resection pending there are no intraoperative factors (i.e., bleeding or hypotension) that change the overall risk of the operation. This individualized strategy can be modified up to the date of the operation or even intraoperatively. Third, the "symptoms" from the colorectal primary do not define the strategy, but rather specific primary tumor factors should be assessed. This includes whether or not the tumor is anatomically obstructive. The authors' institutional practice is that the reverse approach is contraindicated in patients in whom an adult colonoscope cannot traverse the tumor. In this scenario, either resection of the primary or colostomy should be performed first. Patients with anemia should be managed with transfusion and chemotherapy rather than upfront surgical resection for "symptomatic" disease. In cases of tumor perforation, surgical therapy must be first priority. However, in most patients presenting with SCRLM, chemotherapy should be administered first.

We present two cases of SCRLM to demonstrate the use of this treatment algorithm, highlighting the utility of the liver-first strategy in patients with a complex presentation. The first case is a patient who presented with an asymptomatic primary tumor of the sigmoid colon and two metastatic liver lesions in segments $4 / 8$ and 6 , with 

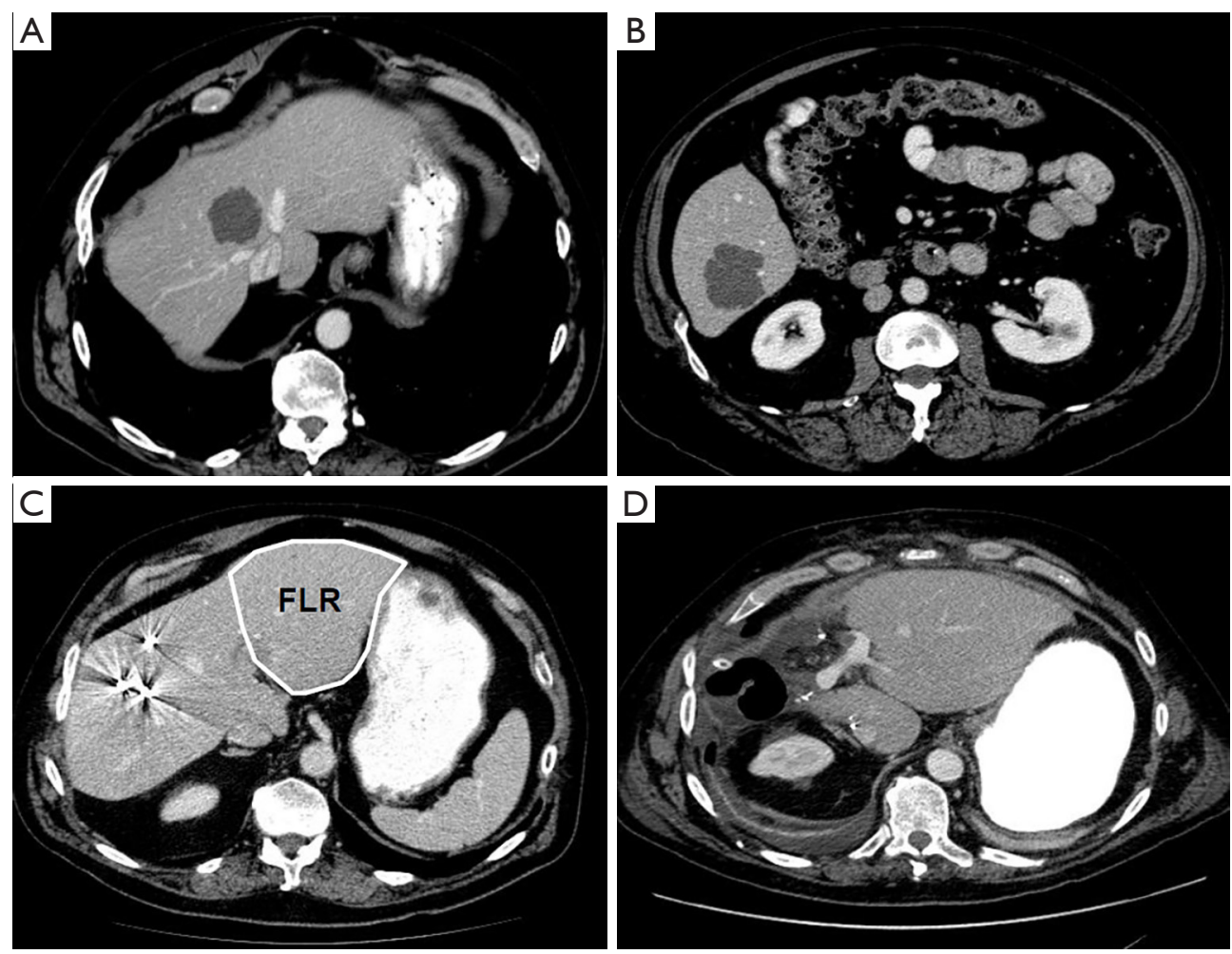

Figure 3 Computed tomography (CT) images from a patient with asymptomatic colon cancer and multiple synchronous liver metastases. $(\mathrm{A}, \mathrm{B})$ After preoperative chemotherapy with FOLFOX-6 + bevacizumab; (C) after portal vein embolization; (D) after extended right hepatectomy and prior to sigmoid colectomy.

the former encasing the middle hepatic vein (Figure 3). After multidisciplinary discussion, the patient received FOLFOX-6 with bevacizumab, which resulted in a type 1 morphologic response (sharp tumor-liver interface and resolution of the peripheral rim of enhancement) (41). The patient subsequently underwent right-sided PVE, which increased the FLR from $10 \%$ to $33 \%$ and allowed for an extended right hepatectomy to be safely performed. The patient underwent a sigmoid colectomy 7 weeks later. Here, using the reverse approach allowed for initial management of systemic disease with early preoperative chemotherapy and avoided delays in approaching the hepatic disease, while ensuring treatment of an asymptomatic primary tumor in a safe fashion.

The second case is a patient presenting with symptoms of obstruction from a primary rectal tumor and synchronous multiple, bilobar metastases (Figure 4). While some may argue this is a patient who should undergo a traditional staged approach, his treatment sequence included a combined first-stage left partial hepatectomy and colostomy. He underwent PVE after 3 weeks and second-stage extended right hepatectomy. After receiving postoperative chemotherapy and external beam radiation therapy, proctectomy was performed. The patient is alive without recurrence 6 years later.

\section{Conclusions}

Patients presenting with CRC and synchronous disease of the liver are a complex group, given the high burden of disease and the variability in presentation. Therefore, the authors recommend a multidisciplinary discussion for all patients with SCRLM. The three recognized surgical strategies for SCRLM include the traditional staged approach, combined approach, and liver-first reverse approach. In recent years, the combined and reverse approaches are being used more often and the authors recommend the use of the traditional staged strategy only for those patients with a primary tumor causing obstruction. Most patients with SCRLM should initially be treated with 

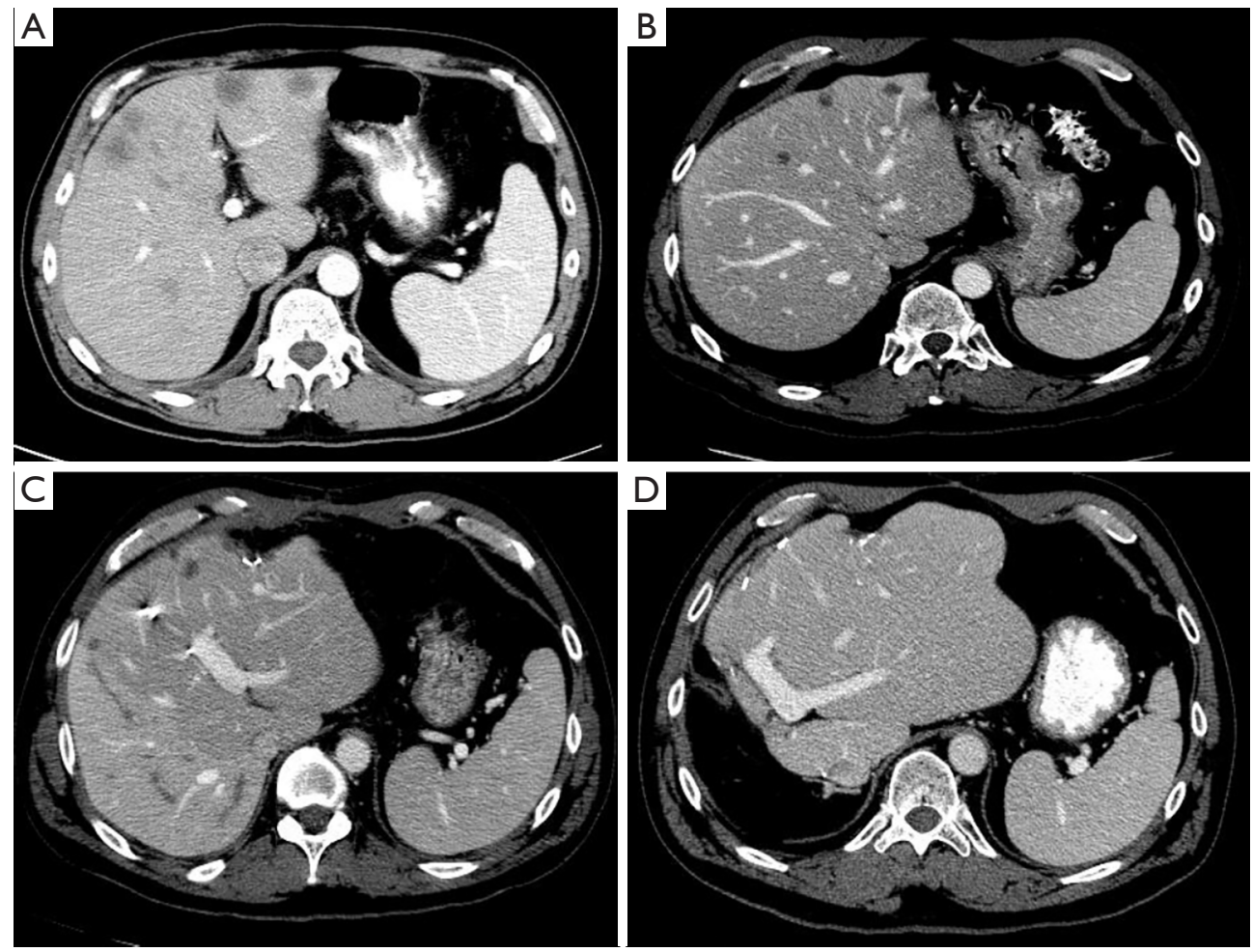

Figure 4 Computed tomography (CT) images from a patient with an obstructive rectal cancer and multiple, bilobar synchronous liver metastases. (A) Pre-treatment CT showing multiple bilobar liver metastases; (B) post-chemotherapy type I morphologic response; (C) after partial left hepatectomy, colostomy, and subsequent right-sided portal vein embolization; (D) after extended right hepatectomy and prior to management of rectal tumor.

preoperative chemotherapy. Importantly, an individualized approach must be taken for all SCRLM patients.

\section{Acknowledgments}

The authors thank Ruth J. Haynes (Department of Surgical Oncology, MD Anderson Cancer Center) for administrative assistance in the preparation of the manuscript.

Funding: Dr. HA Lillemoe is supported by National Institutes of Health grant T32CA009599 and the MD Anderson Cancer Center support grant P30 CA016672.

\section{Footnote}

Conflicts of Interest: The authors have no conflicts of interest to declare.

Ethical Statement: The authors are accountable for all aspects of the work in ensuring that questions related to the accuracy or integrity of any part of the work are appropriately investigated and resolved.

\section{References}

1. World Health Organization (WHO) News: Cancer. Available online: https://www.who.int/news-room/factsheets/detail/cancer, accessed February 14. 2019.

2. Jegatheeswaran S, Mason JM, Hancock HC, et al. The liver-first approach to the management of colorectal cancer with synchronous hepatic metastases: a systematic review. JAMA Surg 2013;148:385-91.

3. Brouquet A, Mortenson MM, Vauthey JN, et al. Surgical strategies for synchronous colorectal liver metastases in 156 consecutive patients: Classic, combined or reverse strategy? J Am Coll Surg 2010;210:934-41.

4. Manfredi S, Lepage C, Hatem C, et al. Epidemiology and management of liver metastases from colorectal cancer. Ann Surg 2006;244:254-9.

5. Conrad C, You N, Vauthey JN. In patients with colorectal liver metastases, can we still rely on number to define 
treatment and outcome? Oncology (Williston Park) 2013;27:1078, 1083-4,11086.

6. Adam R, de Gramont A, Figueras J, et al. Managing synchronous liver metastases from colorectal cancer: A multidisciplinary international consensus. Cancer Treat Rev 2015;41:729-41.

7. Fong Y, Fortner J, Sun RL, et al. Clinical score for predicting recurrence after hepatic resection for metastatic colorectal cancer: analysis of 1001 consecutive cases. Ann Surg 1999;230:309-18.

8. Scheele J, Stang R, Altendorf-Hofmann A, et al. Resection of colorectal liver metastases. World J Surg 1995;19:59-71.

9. Abdalla EK, Vauthey JN, Ellis LM, et al. Recurrence and outcomes following hepatic resection, radiofrequency ablation, and combined resection/ablation for colorectal liver metastases. Ann Surg 2004;239:818-25.

10. Choti MA, Sitzmann JV, Tiburi MF, et al. Trends in long-term survival following liver resection for hepatic colorectal metastases. Ann Surg 2002;235:759-66.

11. Wanis KN, Pineda-Solis K, Tun-Abraham ME, et al. Management of colorectal cancer with synchronous liver metastases: impact of multidisciplinary case conference review. Hepatobiliary Surg Nutr 2017;6:162-9.

12. Reddy SK, Barbas AS, Clary BM. Synchronous colorectal liver metastases: is it time to reconsider traditional paradigms of management? Ann Surg Oncol 2009;16:2395-410.

13. Tebbutt NC, Norman AR, Cunningham D, et al. Intestinal complications after chemotherapy for patients with unresected primary colorectal cancer and synchronous metastases. Gut 2003;52:568-73.

14. Ruo L, Gougoutas C, Paty PB, et al. Elective bowel resection for incurable stage IV colorectal cancer: prognostic variables for asymptomatic patients. J Am Coll Surg 2003;196:722-8.

15. Benoist S, Pautrat K, Mitry E, et al. Treatment strategy for patients with colorectal cancer and synchronous irresectable liver metastases. Br J Surg 2005;92:1155-60.

16. Poultsides GA, Servais EL, Saltz LB, et al. Outcome of primary tumor in patients with synchronous stage IV colorectal cancer receiving combination chemotherapy without surgery as initial treatment. J Clin Oncol 2009;27:3379-84.

17. McCahill LE, Yothers G, Sharif S, et al. Primary mFOLFOX6 plus bevacizumab without resection of the primary tumor for patients presenting with surgically unresectable metastatic colon cancer and an intact asymptomatic colon cancer: definitive analysis of NSABP trial C-10. J Clin Oncol 2012;30:3223-8.

18. Scoggins CR, Meszoely IM, Blanke CD, et al. Nonoperative management of primary colorectal cancer in patients with stage iV disease. Ann Surg Oncol 1999;6:651-7.

19. Bismuth H, Adam R, Levi F, et al. Resection of nonresectable liver metastases from colorectal cancer after neoadjuvant chemotherapy. Ann Surg 1996;224:509-20.

20. Abdalla EK, Adam R, Bilchik AJ, et al. Improving resectability of hepatic colorectal metastases: Expert consensus statement. Ann Surg Oncol 2006;13:1271-80.

21. Loupakis F, Cremolini C, Masi G, et al. Initial therapy with FOLFOXIRI and bevacizumab for metastatic colorectal cancer. N Engl J Med 2014;371:1609-18.

22. Heinemann V, von Weikersthal LF, Decker T, et al. FOLFIRI plus cetuximab versus FOLFIRI plus bevacizumab as first-line treatment for patients with metastatic colorectal cancer (FIRE-3): a randomised, open-label, phase 3 trial. Lancet Oncol 2014;15:1065-75.

23. Venook AP, Niedzwiecki D, Lenz H-J, et al. CALGB/ SWOG 80405: Phase III trial of irinotecan/5-FU/ leucovorin (FOLFIRI) or oxaliplatin/5-FU/leucovorin (mFOLFOX6) with bevacizumab (BV) or cetuximab (CET) for patients (pts) with KRAS wild-type (wt) untreated metastatic adenocarcinoma of the colon or rectum (MCRC). J Clin Oncol 2014;32:15_suppl LBA3.

24. Nordlinger B, Sorbye H, Glimelius B, et al. Perioperative chemotherapy with FOLFOX4 and surgery versus surgery alone for resectable liver metastases from colorectal cancer (EORTC Intergroup trial 40983): a randomised controlled trial. Lancet 2008;371:1007-16.

25. Nordlinger B, Sorbye H, Glimelius B, et al. Perioperative FOLFOX 4 chemotherapy and surgery versus surgery alone for resectable liver metastases from colorectal cancer (EORTC 40983): long-term results of a randomised, controlled, phase 3 trial. Lancet Oncol 2013;14:1208-15.

26. Mitry E, Fields ALA, Bleiberg H, et al. Adjuvant chemotherapy after potentially curative resection of metastases from colorectal cancer: A pooled analysis of two randomized trials. J Clin Oncol 2008;26:4906-11.

27. Ychou M, Hohenberger W, Thezenas S, et al. A randomized phase III study comparing adjuvant 5 -fluorouracil/folinic acid with FOLFIRI in patients following complete resection of liver metastases from colorectal cancer. Annals of Oncology 2009;20:1964-70.

28. Portier G, Elias D, Bouche O, et al. Multicenter randomized trial of adjuvant fluorouracil and folinic acid 
compared with surgery alone after resection of colorectal liver metastases: FFCD ACHBTH AURC 9002 Trial. J Clin Oncol 2006;24:4976-82.

29. Benson AB, Venook AP, Al-Hawary MM, et al. NCCN Guidelines Insights: Colon Cancer, Version 2.2018. J Natl Compr Canc Netw 2018;16:359-69.

30. Vauthey JN, Pawlik TM, Ribero D, et al. Chemotherapy regimen predicts steatohepatitis and an increase in 90-day mortality after surgery for hepatic colorectal metastases. J Clin Oncol 2006;24:2065-72.

31. Kooby DA, Fong Y, Suriawinata A, et al. Impact of steatosis on perioperative outcome following hepatic resection. J Gastrointest Surg 2003;7:1034-44.

32. Nakano H, Oussoultzoglou E, Rosso E, et al. Sinusoidal injury increases morbidity after major hepatectomy in patients with colorectal liver metastases receiving preoperative chemotherapy. Ann Surg 2008;247:118-24.

33. Nordlinger B, Guiguet M, Vaillant JC, et al. Surgical resection of colorectal carcinoma metastases to the liver: A prognostic scoring system to improve case selection, based on 1568 patients. Cancer 1996;77:1254-62.

34. Bolton JS, Fuhrman GM. Survival after resection of multiple bilobar hepatic metastases from colorectal carcinoma. Ann Surg 2000;231:743-51.

35. Scheele J, Stangl R, Altendorf-Hofmann A, et al. Indicators of prognosis after hepatic resection for colorectal secondaries. Surgery 1991;110:13-29.

36. Vogt P, Raab R, Ringe B, et al. Resection of synchronous liver metastases from colorectal cancer. World J Surg 1991;15:62-7.

37. Bismuth H, Castaing D, Traynor O. Surgery for synchronous hepatic metastases of colorectal cancer. Scand J Gastroenterol Suppl 1988;149:144-9.

38. Martin R, Paty P, Fong Y, et al. Simultaneous liver and colorectal resections are safe for synchronous colorectal liver metastasis. J Am Coll Surg 2003;197:233-41.

39. Reddy SK, Pawlik TM, Zorzi D, et al. Simultaneous resections of colorectal cancer and synchronous liver metastases: A multi-institutional analysis. Ann Surg Oncol 2007;14:3481-91.

40. Mentha G, Majno PE, Andres A, et al. Neoadjuvant chemotherapy and resection of advanced synchronous liver metastases before treatment of the colorectal primary. Br J Surg 2006;93:872-8.

41. Chun YS, Vauthey JN, Boonsirikamchai P, et al. Association of computed tomography morphologic criteria with pathologic response and survival in patients treated with bevacizumab for colorectal liver metastases. JAMA
2009;302:2338-44.

42. Shindoh J, Loyer EM, Kopetz S, et al. Optimal morphologic response to preoperative chemotherapy: an alternate outcome end point before resection of hepatic colorectal metastases. J Clin Oncol 2012;30:4566-72.

43. Suzuki K, Muto Y, Ichida K, et al. Morphological response contributes to patient selection for rescue liver resection in chemotherapy patients with initially unresectable colorectal liver metastasis. Oncology letters 2017;14:1491-9.

44. Pawlik TM, Olino K, Gleisner AL, et al. Preoperative chemotherapy for colorectal liver metastases: impact on hepatic histology and postoperative outcome. J Gastrointest Surg 2007;11:860-8.

45. Kawaguchi Y, Lillemoe HA, Vauthey JN. Dealing with an insufficient future liver remnant: Portal vein embolization and two-stage hepatectomy. J Surg Oncol 2019.

46. Jaeck D, Oussoultzoglou E, Rosso E, et al. A two-stage hepatectomy procedure combined with portal vein embolization to achieve curative resection for initially unresectable multiple and bilobar colorectal liver metastases. Ann Surg 2004;240:1037-49.

47. Adam R, Laurent A, Azoulay D, et al. Two-stage hepatectomy: A planned strategy to treat irresectable liver tumors. Ann Surg 2000;232:777-85.

48. Brouquet A, Abdalla EK, Kopetz S, et al. High survival rate after two-stage resection of advanced colorectal liver metastases: response-based selection and complete resection define outcome. J Clin Oncol 2011;29:1083-90.

49. Narita M, Oussoultzoglou E, Jaeck D, et al. Twostage hepatectomy for multiple bilobar colorectal liver metastases. Br J Surg 2011;98:1463-75.

50. Passot G, Chun YS, Kopetz SE, et al. Predictors of safety and efficacy of 2-stage hepatectomy for bilateral colorectal liver metastases. J Am Coll Surg 2016;223:99-108.

51. Blazer DG, 3rd, Kishi Y, Maru DM, et al. Pathologic response to preoperative chemotherapy: a new outcome end point after resection of hepatic colorectal metastases. J Clin Oncol 2008;26:5344-51.

52. Maru DM, Kopetz S, Boonsirikamchai P, et al. Tumor thickness at the tumor-normal interface: a novel pathologic indicator of chemotherapy response in hepatic colorectal metastases. Am J Surg Pathol 2010;34:1287-94.

53. Vauthey JN, Zimmitti G, Kopetz SE, et al. RAS mutation status predicts survival and patterns of recurrence in patients undergoing hepatectomy for colorectal liver metastases. Ann Surg 2013;258:619-26.

54. Margonis GA, Spolverato G, Kim Y, et al. Effect of KRAS 
mutation on long-term outcomes of patients undergoing hepatic resection for colorectal liver metastases. Ann Surg Oncol 2015;22:4158-65.

55. Lillemoe HA, Kawaguchi Y, Passot G, et al. Surgical resection for recurrence after two-stage hepatectomy for colorectal liver metastases is feasible, is safe, and improves survival. J Gastrointest Surg 2019;23:84-92.

56. Brudvik KW, Kopetz SE, Li L, et al. Meta-analysis of KRAS mutations and survival after resection of colorectal liver metastases. Br J Surg 2015;102:1175-83.

57. Yokota T, Ura T, Shibata N, et al. BRAF mutation is

Cite this article as: Lillemoe HA, Vauthey JN. Surgical approach to synchronous colorectal liver metastases: staged, combined, or reverse strategy. Hepatobiliary Surg Nutr 2020;9(1):25-34. doi: 10.21037/hbsn.2019.05.14 a powerful prognostic factor in advanced and recurrent colorectal cancer. Br J Cancer 2011;104:856-62.

58. Tran B, Kopetz S, Tie J, et al. Impact of BRAF mutation and microsatellite instability on the pattern of metastatic spread and prognosis in metastatic colorectal cancer. Cancer 2011;117:4623-32.

59. Schirripa M, Bergamo F, Cremolini C, et al. BRAF and RAS mutations as prognostic factors in metastatic colorectal cancer patients undergoing liver resection. Br J Cancer 2015;112:1921-8. 